\title{
Perception des producteurs sur l'utilisation des déjections de chenilles dans la gestion de la fertilité des sols dans la région des Hauts-Bassins au Burkina Faso
}

Coulibaly Kalifa ${ }^{13}$, Gnissien Moussa1, Yaméogo T. Jérôme², Traoré Mamadou1, Nacro B. Hassan1 1 Laboratoire d'étude et de recherche sur la fertilité du sol (LERF), Institut du Développement Rural (IDR), Université Polytechnique de Bobo-Dioulasso (UPB) Bobo-Dioulasso ; BP 1091, Bobo-Dioulasso, Burkina Faso.

'Laboratoire des systèmes naturels, des agrosystèmes et de l'ingénierie de l'environnement (SyNAIE), Institut du Développement Rural (IDR), Université Polytechnique de Bobo-Dioulasso (UPB) Bobo-Dioulasso ; BP 1091, BoboDioulasso, Burkina Faso.

${ }^{3}$ Unité de Recherche sur les Productions Animales (URPAN), Centre International de Recherche-Développement sur l'Elevage en zone Sub-humide (CIRDES), BP 454, Bobo-Dioulasso, Burkina Faso.

*Auteur correspondant: kalifacoul1@yahoo.fr

Original submitted in on $16^{\text {th }}$ November 2016. Published online at www.m.elewa.org on $31^{\text {st }}$ December 2016 http://dx.doi.org/10.4314/jab.v108i1.6

\section{RESUME}

Objectif : L'étude vise à analyser la perception des producteurs sur la contribution des chenilles dans la gestion de la fertilité des sols et dans la production des cultures.

Méthodologie et résultats : Les enquêtes ont été effectuées auprès de 51 producteurs dans 5 villages de la région des Hauts-Bassins (Burkina Faso) qui se caractérisent par l'importance des chenilles. Les données collectées, ont concerné les caractéristiques des exploitations, les perceptions des producteurs sur le comportement des chenilles de karité et l'effet des déjections de chenilles sur la fertilité du sol et la production des cultures. Cinq groupes de producteurs ont été identifiés et les caractéristiques et perceptions ont été analysées. La superficie des champs, l'âge des producteurs et la densité de karité sont les facteurs qui discriminent les producteurs.

Conclusions et application des résultats : Les producteurs enquêtés estiment à plus de $90 \%$ que les chenilles apparaissent à la même période de chaque année. Entre 57 et $100 \%$ des producteurs déclarent que les chenilles apparaissent chaque année. Les producteurs enquêtés ont par ailleurs, identifié les facteurs anthropiques, climatiques et édaphiques comme les facteurs pouvant influencer la dynamique des chenilles suivant les années. Les déjections de chenilles sont perçues par les producteurs comme fertilisants pouvant contribuer à améliorer la production des cultures. La collecte manuelle des déjections de chenille est donc proposée par près de $50 \%$ des producteurs qui militent pour l'épandage et l'apport localisé comme modes d'application des déjections. Cette perception des producteurs doit conduire à la réalisation de recherches sur l'effet des déjections sur le sol et les cultures, et à la promotion des déjections de chenilles comme mode de gestion écologique de la fertilité des sols dans les parcs à karité.

Mots clés : parc à karité, déjection de chenille, fertilisation, agro-écologie, Burkina Faso. 
ABSTRACT

Perception of the producers on the use of caterpillar droppings in soil fertility management in Burkina Faso

Objective : The study aims to analyze the perception of the producers on the contribution of caterpillars in the management of soil fertility and crop production.

Methods and Results: The surveys were conducted with 51 farmers in 5 villages in the Hauts-Bassins (Burkina Faso) which is characterized by the importance of the caterpillars. The data collected were for farm characteristics, perceptions of the producers on the behavior of caterpillars Shea and the effect of caterpillars manure on soil fertility and crop production. Five producer groups have been identified and the characteristics and perceptions were analyzed. Field size, age of producers and shea density are the factors that discriminate producers.

Conclusions and application of results : Respondents producers estimate that over $90 \%$ of the caterpillars appear in the same period of each year. Between 57 and $100 \%$ of producers say that the caterpillars emerge each year. Respondents producers have also identified the anthropic, climate and soil as factors influencing the dynamics of caterpillars following years. Caterpillar droppings are perceived by farmers as fertilizer that can improve crop production. The manual collection of caterpillar droppings is proposed by nearly $50 \%$ of producers who campaign for spreading and providing localized as modes of application of manure. This perception of producers should lead to the realization of research on the effect of manure on the soil and crops and promotion of caterpillar droppings as ecological management of soil fertility in shea parks.

Keywords : Shea Park, caterpillar droppings, fertilizer, yield, agro-ecology

\section{INTRODUCTION GENERALE}

Les arbres épargnés dans les champs, permettent de constituer des parcs agroforestiers dans la plupart des pays sahéliens et subhumides de l'Afrique de l'Ouest. Les arbres sont épargnés pour servir de source d'aliments, de combustible, de produits médicinaux, de maintien de la fertilité des sols et la protection de l'environnement (Boffa, 2000). Les parcs à karité (Vitellaria paradoxa Gaertn. f.) et à néré (Parkia biglobosa (Jacq.) R. $\mathrm{Br}$. ex $\mathrm{G}$ Don) sont les plus répandus dans les zones semi-arides de l'Afrique de l'Ouest (Boffa, 2000 ; Kaboré et al., 2012). Si le karité produit des amendes qui sont beaucoup utilisées pour la production de beurre, il faut noter que ses feuilles constituent une source alimentaire pour certains insectes notamment les chenilles de karité "Cirina butyrospermi Vuillet". Au Burkina Faso, l'aire de répartition de la chenille de karité s'est rétrécie. Les foyers les plus importants, se situent dans les régions des Hauts-Bassins et des Cascades. Les chenilles présentent de grandes valeurs nutritionnelles si bien qu'elles peuvent faire l'objet " d'agrobussiness » et de "médico-business " (Sanon, 2005). Les travaux de Ouédraogo (1993) ont montré que la chenille de karité est un aliment riche et contient jusqu'à $63 \%$ de protéines, $15 \%$ de matière grasse, $0,16 \%$ de calcium, $2,25 \%$ de potassium et de la vitamine B12. Compte tenu de leur richesse en protéines, elles sont conseillées pour lutter contre la malnutrition chez les enfants à bas âge (Sanon, 2005). La cueillette des chenilles constitue une activité génératrice de revenus à différents niveaux. Selon une étude de Sermé (2011) sur le circuit de commercialisation des chenilles dans la province du Houet, la cueillette des chenilles rapportait une marge nette estimée à environ 172825 FCFA, 748970 FCFA et environ 2133432 FCFA respectivement pour le producteur/cueilleur, le détaillant et le grossiste sur une campagne. En plus donc de leur importance nutritionnelle et économique, les chenilles en consommant les feuilles de karité réduisent voire éliminent sur une période de culture l'ombrage dû aux feuilles et rejettent également des déjections riches en éléments fertilisants (Coulibaly et al., 2016). L'objectif de cette étude était de montrer la contribution des chenilles dans la gestion de la fertilité des sols et dans la production des cultures à travers une analyse de la perception des producteurs. 


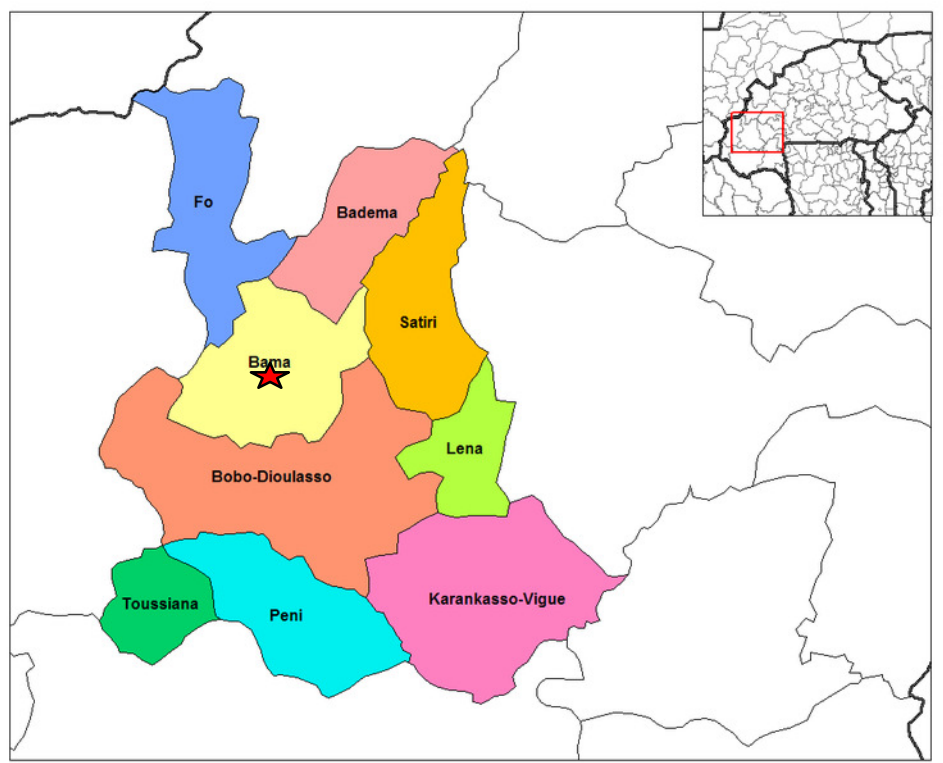

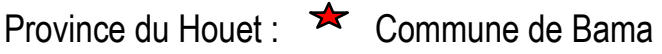

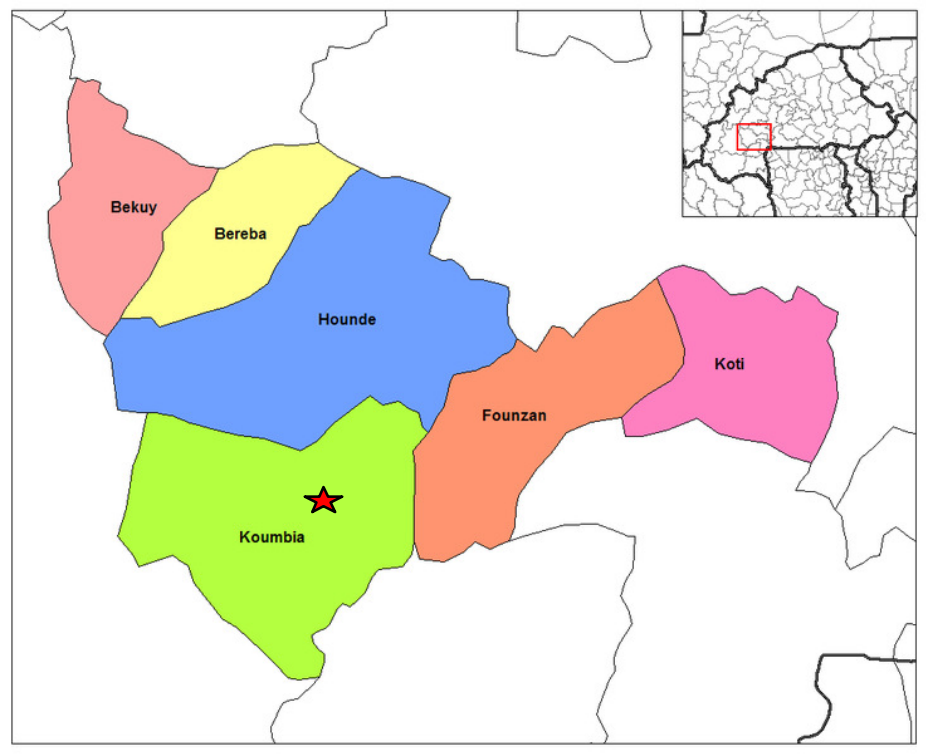

Province du Tuy : $\approx$ Commune de Koumbia

Figure 1 : Cartes des sites d'étude (source : https://fr.wikipedia.org/)

\section{MATERIEL ET METHODES}

Présentation de la zone d'étude : L'étude a eu lieu dans deux communes rurales de la région des HautsBassins (Burkina Faso). II s'est agi des communes rurales de Bama et de Koumbia. Les coordonnées de la commune rurale de Bama sont $11^{\circ} 22^{\prime} 04^{\prime \prime}$ de latitude Nord et $4^{\circ} 25^{\prime} 12^{\prime \prime}$ de longitude Ouest et une altitude de $300 \mathrm{~m}$. Les coordonnées de la commune rurale de Koumbia sont: $4^{\circ} 24^{\prime} 01^{\prime \prime} \mathrm{de}$ longitude Ouest, $12^{\circ} 42^{\prime} 20$ "de latitude Nord et une altitude $290 \mathrm{~m}$ (Blanchard, 2005). Dans la commune rurale de Bama, deux villages (Banankélédaga et Desso) ont été concernés par l'étude. Au niveau de la commune rurale de Koumbia, l'étude s'est déroulée dans trois villages
(Makognadougou, Dougoumato 1 et Dougoumato 2). La commune rurale de Bama appartient au climat sudsoudanien avec des moyennes pluviométriques annuelles comprises entre 1100 et $1200 \mathrm{~mm}$ (Fontès et Guinko, 1995). Le climat est caractérisé par deux saisons distinctes : une saison pluvieuse de 4 à 5 mois (mai à septembre) durant laquelle souffle la mousson et une saison sèche (allant d'octobre à avril) où souffle I'harmattan. La végétation de la commune rurale de Bama selon Fontès et Guinko (1995) est celle de la savane boisée. Elle peut être divisée en trois strates: arborée, arbustive et herbacée. La strate arborée comporte des espèces comme Vittelaria paradoxa, 
Parkia biglobosa, Tamarindus indica L. La strate arbustive est constituée par les Combretacées et des espèces comme Piliostigma thonningii (Schumach.) Milne-Redh, P. reticulatum (DC.) Hochstet, Daniellia oliveri (Rolfe) Hutch. et Dalz, qui peuple les jachères. La commune rurale de Koumbia appartient au domaine climatique de type Nord-soudanien avec une pluviométrie annuelle variant entre 800 et $1100 \mathrm{~mm}$ (Fontès et Guinko, 1995). Le climat est caractérisé par deux saisons : une saison pluvieuse pendant laquelle souffle la mousson d'une durée de 4 mois centrée sur les mois de juillet et Août et une saison sèche (allant d'octobre à avril) où l'harmattan est le vent dominant. La végétation de la commune de Koumbia se compose de savanes arbustives et arborées à la périphérie des terroirs ou le long des cours d'eau. Les centres des territoires villageois sont occupés par les champs mis en culture ou en jachère. La forêt classée de la Mou, située du côté Est du terroir de Koumbia, présente par endroits des forêts denses à Cola cordifolia (Cav) R.Br.et Terminalia laxiflora Engl.et des forêts claires à Gardenia erubescens Stapf \& Hutch. et Daniella oliveri (Blanchard, 2005). La Error! Reference source not found. présente les cartes des sites d'étude.

Echantillonnage et collecte des données: Les données ont été collectées dans la région des HautsBassins du fait de l'importance des chenilles dans cette localité du Burkina Faso. Les communes concernées par l'étude sont Koumbia dans la province du Tuy et Bama dans la province du Houet. Les 2 communes se distinguent par l'importance de la culture du coton à Koumbia et l'importance de la culture du riz et du maraîchage à Bama. Les enquêtés ont été choisis au

\section{RESULTATS}

Caractéristiques des exploitations enquêtées et constitution de classes homogènes : Le Tableau 1 montre que les producteurs enquêtés ont un âge compris entre 22 et 65 ans avec une moyenne de 42 ans. Les exploitations des producteurs interviewés ont un nombre moyen de 10 personnes avec 6 actifs en moyenne. Ces exploitations on 3 champs en moyenne avec une surface totale de 12 ha. Les principaux champs des exploitations ont un âge moyen de mise en culture de 21 ans avec une superficie de 5 ha et une densité de karité de 16 pieds/ha. La classification ascendante hiérarchique (CAH) donne 5 classes de producteurs avec une coupure du dendrogramme à un niveau de dissimilarité 27 (Figure 2). L'analyse des caractéristiques de ces classes (

Tableau 2), montre que la classe $1(n=14)$ se compose de producteurs jeunes ( $36 \pm 5$ ans) ayant peu hasard dans les villages. Au total, 51 producteurs ont été enquêtés dont 10 producteurs par village pour les villages de Makognadougou, Dougoumato 2, Banakélédaga, Desso et 11 producteurs pour le village de Dougoumato 1. Une fiche d'enquête individuelle a été établie pour effectuer les interviews. Les données collectées auprès de chaque producteur ont concerné les caractéristiques des exploitations (âge du producteur, nombre de personnes et d'actifs, taille des parcelles de cultures, densité de karité, équipement en traction animale), les perceptions sur le comportement des chenilles de karité (période et fréquence d'apparition, dégât sur le karité et les cultures) et les perceptions sur l'effet des déjections de chenilles sur la fertilité du sol et la production des cultures.

Traitement statistique des données: La méthode d'analyse multi-variée a été utilisée du fait qu'il y a une diversité de réponses aux questions. Le logiciel XLSTAT 2015.4.01.21575a été utilisé pour réaliser une analyse en composantes principales (ACP) sur les données liées aux caractéristiques des exploitations (âge du chef d'exploitation, nombre de personnes et d'actifs de l'exploitation, nombre de champs, superficie totale des champs, surface et âge de mise en culture du champ principal et densité de karité sur le champ principal). Une classification ascendante hiérarchique (CAH) a ensuite été réalisée à partir du tableau des coordonnées des observations sur les axes factoriels obtenus de l'ACP. Elle a permis de constituer des groupes homogènes de producteurs. Le logiciel Microsoft Excel 2010 a été utilisé pour générer les tableaux et les graphiques.

de personnes ( $7 \pm 2$ personnes) et d'actifs ( $4 \pm 1$ actifs) dans leurs exploitations. Ces jeunes producteurs ont un champ principal de petite surface ( $3 \pm 2$ ha) avec un âge de mise en culture plus jeune (12 \pm 6 ans). La classe 1 est composée de $29 \%$ de producteurs de la commune de Koumbia et $71 \%$ de ceux de Bama. La classe $2(n=24)$ se caractérise de producteurs dont l'âge moyen est de $43 \pm 9$ ans avec peu de champs (2 \pm 1 champs) de surface totale moyenne de $8 \pm 4 \mathrm{ha}$. La classe 2 est composée de $83 \%$ de producteurs de la commune de Koumbia et $17 \%$ de ceux de Bama. La classe $3(n=07)$ se compose de producteurs plus âgés (59 \pm 5 ans) que toutes les autres classes. Les producteurs de cette classe 3 ont en moyenne $4 \pm 1$ ha de champ dont l'âge moyen de mise en culture du principal champ est de $35 \pm 22$ ans avec une densité importante de karité ( $26 \pm 33$ pieds/ha). La classe 3 est composée de $14 \%$ de producteurs de la commune de Koumbia et $86 \%$ de ceux de Bama. La classe 4 se compose de 3 producteurs qui ont un âge moyen de 42 
\pm 3 ans avec plus de personnes à nourrir ( $28 \pm 10$ personnes) et d'actifs (19 \pm 5 actifs) que les autres classes. La classe 5 se compose également de 3 producteurs jeunes ( $36 \pm 4$ ans) ayant en moyenne $2 \pm$ 1 champs de grandes surfaces ( $53 \pm 41$ ha). Cette classe 5 se caractérise aussi par une faible densité de karité sur le champ principal ( $8 \pm 8$ pieds/ha). Les classes 4 et 5 sont composées uniquement de producteurs de la commune de Koumbia. La Figure 3 montre que la majorité des producteurs ont la traction animale et que d'autres sont toujours en manuel. Les données indiquent que $57,14 \%, 83,33 \%, 42,86 \%$, $66,67 \%$ et $100 \%$ des producteurs enquêtés respectivement des classes 1, 2, 3, 4 et 5 sont équipés en traction animale.

Tableau 1 : Statistiques descriptives de l'échantillon de producteurs enquêtés

\begin{tabular}{lccc}
\hline Variable & Minimum & Moyenne & Maximum \\
\hline Age chef exploitation (an) & 22 & 42 & 65 \\
Nombre de personnes & 4 & 10 & 40 \\
Nombre d'actifs & 2 & 6 & 25 \\
Nombre dechamps & 1 & 3 & 8 \\
STC (ha) & 1 & 12 & 100 \\
SCP (ha) & 0,25 & 5 & 30 \\
Age CP (an) & 3 & 21 & 65 \\
Densité Karité CP (pieds/ha) & 0 & 16 & 100 \\
\hline
\end{tabular}

Légende : $\mathrm{STC}$ = surface totale des champs, $\mathrm{SCP}=$ surface du champ principal, $\mathrm{CP}=$ champ principal

Tableau 2 : Caractéristiques des classes de producteurs enquêtés

\begin{tabular}{|c|c|c|c|c|c|c|}
\hline & & $\begin{array}{c}\text { Classe } 1(n= \\
14)\end{array}$ & $\begin{array}{c}\text { Classe } 2(n= \\
24)\end{array}$ & $\begin{array}{c}\text { Classe } 3(n= \\
07)\end{array}$ & $\begin{array}{c}\text { Classe } 4(n= \\
03)\end{array}$ & $\begin{array}{c}\text { Classe } 5(n= \\
03)\end{array}$ \\
\hline Répartition des & Koumbia (\%) & 29 & 83 & 14 & 100 & 100 \\
\hline $\begin{array}{l}\text { producteurs par } \\
\text { commune }\end{array}$ & Bama (\%) & 71 & 17 & 86 & 0 & 0 \\
\hline Age (an) & & $36 \pm 5$ & $43 \pm 9$ & $59 \pm 5$ & $42 \pm 3$ & $36 \pm 4$ \\
\hline Nombre personn & & $7 \pm 2$ & $10 \pm 4$ & $9 \pm 3$ & $28 \pm 10$ & $11 \pm 4$ \\
\hline Nombre Actifs & & $4 \pm 1$ & $6 \pm 3$ & $8 \pm 3$ & $19 \pm 5$ & $8 \pm 2$ \\
\hline Nombre champs & & $4 \pm 2$ & $2 \pm 1$ & $4 \pm 1$ & $3 \pm 1$ & $2 \pm 1$ \\
\hline Surface totale de & champs (ha) & $13 \pm 17$ & $8 \pm 4$ & $9 \pm 9$ & $10 \pm 5$ & $53 \pm 41$ \\
\hline Surface du cham & principal (ha) & $3 \pm 2$ & $4 \pm 2$ & $4 \pm 2$ & $6 \pm 4$ & $25 \pm 8$ \\
\hline Age champ princi & al (an) & $12 \pm 6$ & $23 \pm 10$ & $35 \pm 22$ & $14 \pm 9$ & $21 \pm 12$ \\
\hline $\begin{array}{l}\text { Densité karité cha } \\
\text { (pieds/ha) }\end{array}$ & np principal & $19 \pm 17$ & $12 \pm 12$ & $26 \pm 33$ & $18 \pm 4$ & $8 \pm 8$ \\
\hline
\end{tabular}

Perceptions des producteurs sur l'apparition des chenilles et leurs dégâts sur les karités et les cultures : La majorité des producteurs de toutes les classes (plus de 90\%), estime que les chenilles apparaissent à la même période chaque année (Tableau 3). II en est de même pour la fréquence d'apparition des chenilles que $57 \%$ (classe 1) à 100\% (classe 5) des producteurs estiment qu'elle est annuelle. Si les chenilles apparaissent à la même période et chaque année, la majorité des producteurs de toutes les classes (plus de 65\%), estime que leur quantité diminue d'année en année. La majorité des producteurs de la classe $1(57,14 \%)$, la classe $3(71,43$ $\%)$ et la classe $4(66,67 \%)$ estime que les chenilles attaquent les feuilles de tous les pieds de karité (Tableau 3). Au niveau des classes 2 et 5, c'est une minorité $(<15 \%)$ qui estime que les chenilles attaquent les feuilles de tous les pieds de karité. On note que 66 à $92 \%$ des producteurs estiment que les chenilles ne nuisent pas au karité. Tous les producteurs (100\%) affirment que les chenilles ne causent pas de dégâts sur les cultures. 


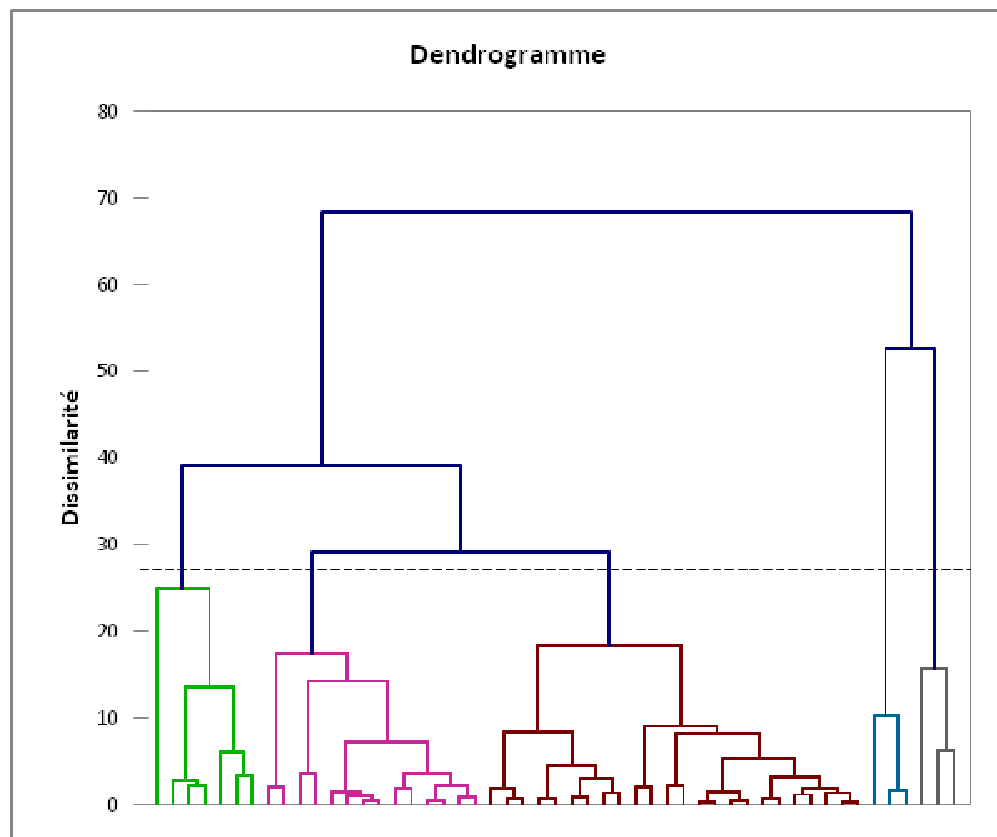

Figure 2 : Classification ascendante hiérarchique des producteurs (Dendrogramme)

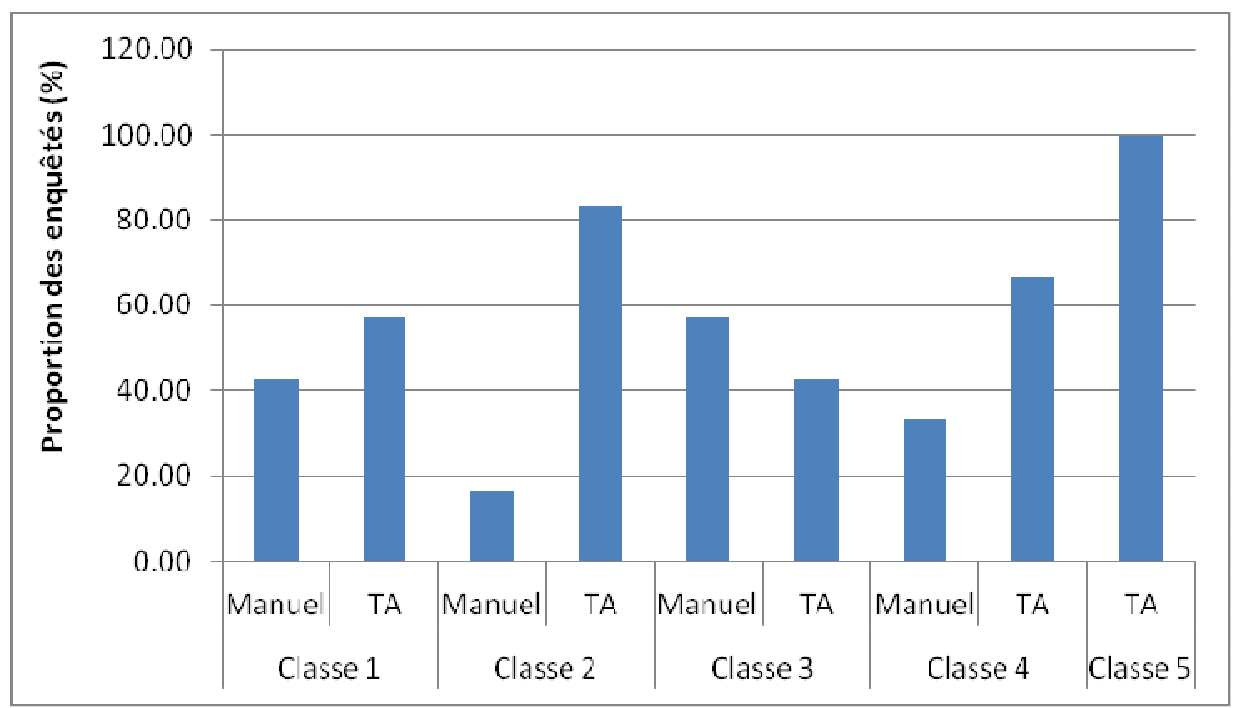

Figure 3 : Equipement en traction animale des exploitations enquêtées ( $T A=$ traction animale)

Tableau 3 : Perceptions des producteurs sur l'apparition des chenilles et leurs dégâts sur les karités et les cultures

\begin{tabular}{|c|c|c|c|c|c|c|c|}
\hline & & $\begin{array}{l}\text { Même } \\
\text { période } \\
\text { apparition }\end{array}$ & $\begin{array}{l}\text { Fréquence } \\
\text { apparition } \\
\text { chenilles }\end{array}$ & $\begin{array}{l}\text { Diminution } \\
\text { Chenilles/an }\end{array}$ & $\begin{array}{l}\text { Touskarité } \\
\text { attaqués }\end{array}$ & $\begin{array}{l}\text { Dégât } \\
\text { sur } \\
\text { Karité }\end{array}$ & $\begin{array}{l}\text { Dégât sur } \\
\text { culture }\end{array}$ \\
\hline Classe 1 & $\begin{array}{l}\text { Non (\%) } \\
\text { Oui (\%) }\end{array}$ & $\begin{array}{l}7,14 \\
92,86\end{array}$ & $\begin{array}{l}42,86 \\
57,14\end{array}$ & $\begin{array}{l}28,57 \\
71,43\end{array}$ & $\begin{array}{l}42,86 \\
57,14\end{array}$ & $\begin{array}{l}92,86 \\
7,14\end{array}$ & $\begin{array}{l}100 \\
0\end{array}$ \\
\hline Classe 2 & $\begin{array}{l}\text { Non (\%) } \\
\text { Oui (\%) }\end{array}$ & $\begin{array}{l}4,17 \\
95,83\end{array}$ & $\begin{array}{l}16,67 \\
83,33\end{array}$ & $\begin{array}{l}33,33 \\
66,67\end{array}$ & $\begin{array}{l}87,50 \\
12,50\end{array}$ & $\begin{array}{l}75,00 \\
25,00\end{array}$ & $\begin{array}{l}100 \\
0\end{array}$ \\
\hline Classe 3 & $\begin{array}{l}\text { Non (\%) } \\
\text { Oui (\%) }\end{array}$ & $\begin{array}{l}0,00 \\
100\end{array}$ & $\begin{array}{l}28,57 \\
71,43\end{array}$ & $\begin{array}{l}28,57 \\
71,43\end{array}$ & $\begin{array}{l}28,57 \\
71,43\end{array}$ & $\begin{array}{l}85,71 \\
14,29\end{array}$ & $\begin{array}{l}100 \\
0\end{array}$ \\
\hline Classe 4 & $\begin{array}{l}\text { Non (\%) } \\
\text { Oui (\%) }\end{array}$ & $\begin{array}{l}0,00 \\
100\end{array}$ & $\begin{array}{l}33,33 \\
66,67\end{array}$ & $\begin{array}{l}33,33 \\
66,67\end{array}$ & $\begin{array}{l}33,33 \\
66,67\end{array}$ & $\begin{array}{l}66,67 \\
33,33\end{array}$ & $\begin{array}{l}100 \\
0\end{array}$ \\
\hline Classe 5 & $\begin{array}{l}\text { Non (\%) } \\
\text { Oui (\%) }\end{array}$ & $\begin{array}{l}0,00 \\
100\end{array}$ & $\begin{array}{l}0 \\
100\end{array}$ & $\begin{array}{l}0,00 \\
100,00\end{array}$ & $\begin{array}{l}100 \\
0\end{array}$ & $\begin{array}{l}66,67 \\
33,33\end{array}$ & $\begin{array}{l}100 \\
0\end{array}$ \\
\hline
\end{tabular}


Perception des producteurs sur les raisons de la diminution des chenilles par an : La Figure 4 montre que $54,55 \%, 41,18 \%$ et $60 \%$ des producteurs respectivement des classes 1,2 et 3 , attribuent la diminution des chenilles aux irrégularités des pluies. Cette diminution est attribuée à l'activité anthropique par $33,33 \%$ et $66,7 \%$ des producteurs respectivement de la classe 4 et 5 . Toutefois, on note que la majorité des producteurs de la classe $2(52 \%)$ et la classe 4 $(66,67 \%)$ n'ont aucune idée concernant les raisons de la diminution des chenilles.

Perception des producteurs sur les effets des chenilles sur la fertilité du sol et la production des cultures : Concernant la fertilité des sols, la majorité des producteurs $(>50 \%$ ) de toutes les classes estime que les sols sous les houppiers attaqués (HA) par les chenilles sont plus fertiles que les sols aussi bien sous houppiers non attaqués $(\mathrm{HnA})$ que hors houppiers $(\mathrm{HH})$. Concernant la production des cultures, $71 \%$ des producteurs des classes 1 et 2 et $100 \%$ des producteurs des classes 3,4 et 5 estiment qu'elle est plus importante sous HA par les chenilles comparés aux HnA. Tous les producteurs des classes 3, 4 et 5 estiment également que la production des cultures est plus importante sous les $\mathrm{HA}$ que $\mathrm{HH}$. Les producteurs des classes 1 et 2, estiment à 64 et $46 \%$ que la production des cultures est plus importante sous les $\mathrm{HA}$ que

$\mathrm{HH}$

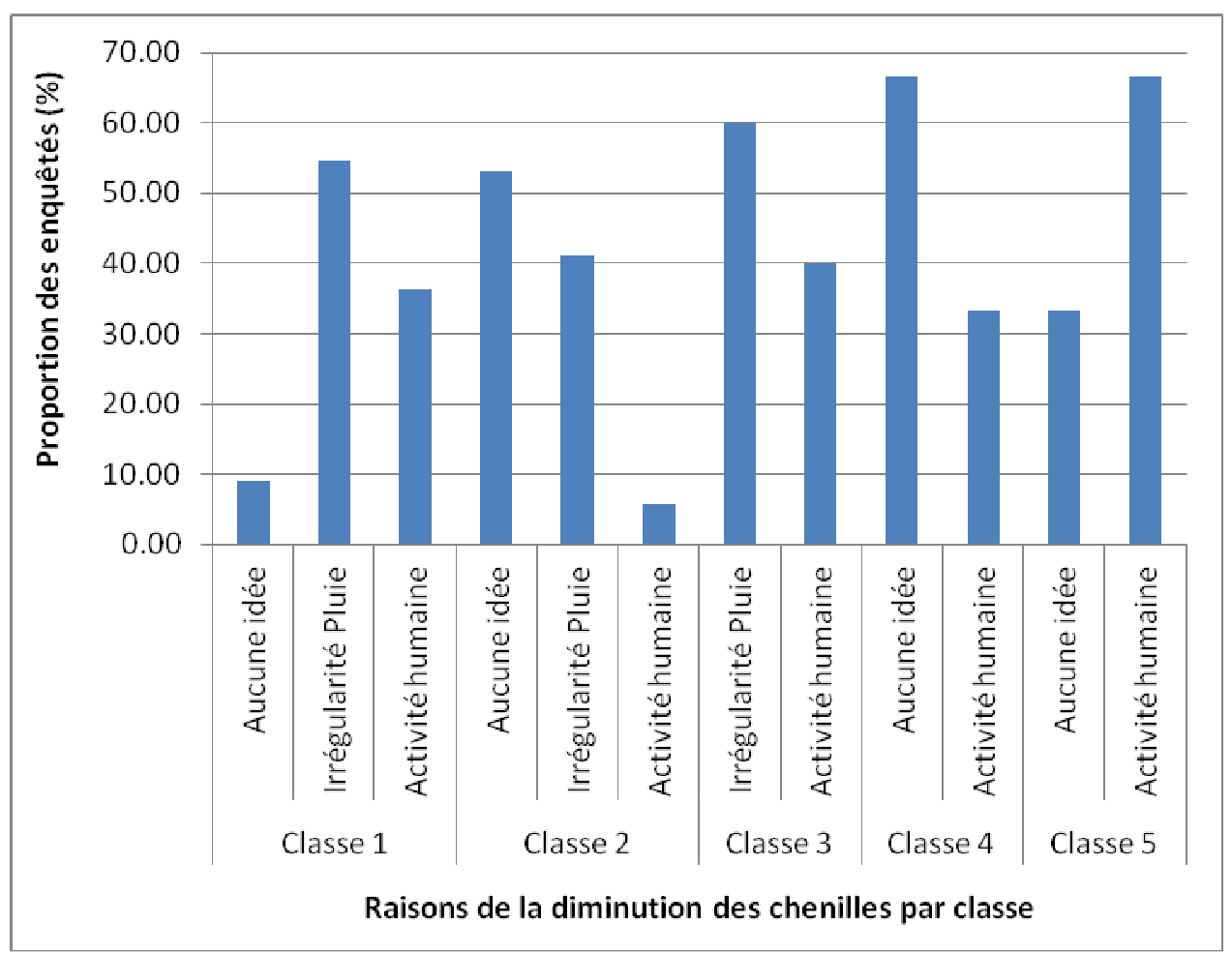

Figure 4: Raisons de la diminution annuelle des chenilles

Tableau 4). 


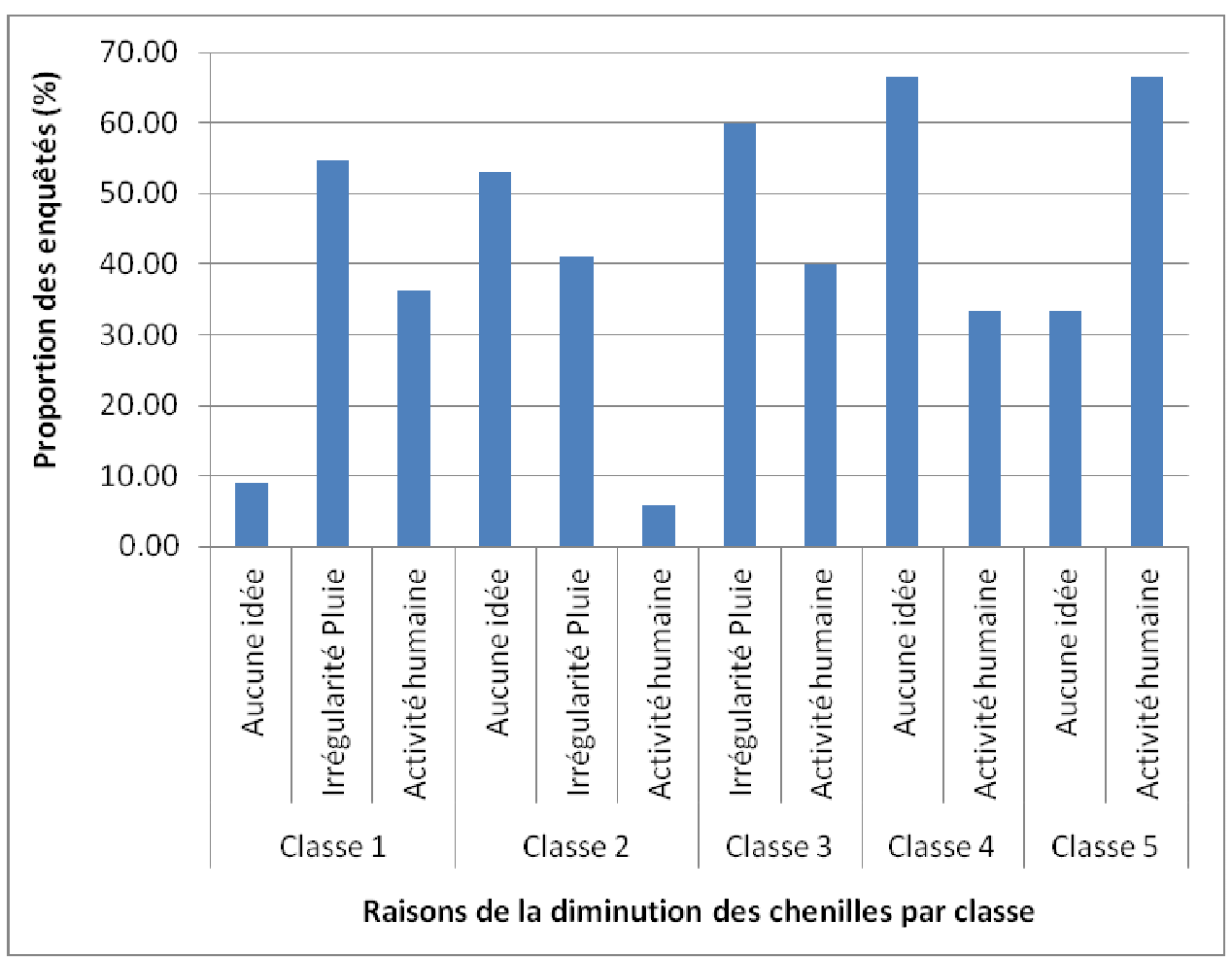

Figure 4 : Raisons de la diminution annuelle des chenilles

Tableau 4 : Perception des producteurs sur les effets des chenilles sur la fertilité du sol et la production des cultures

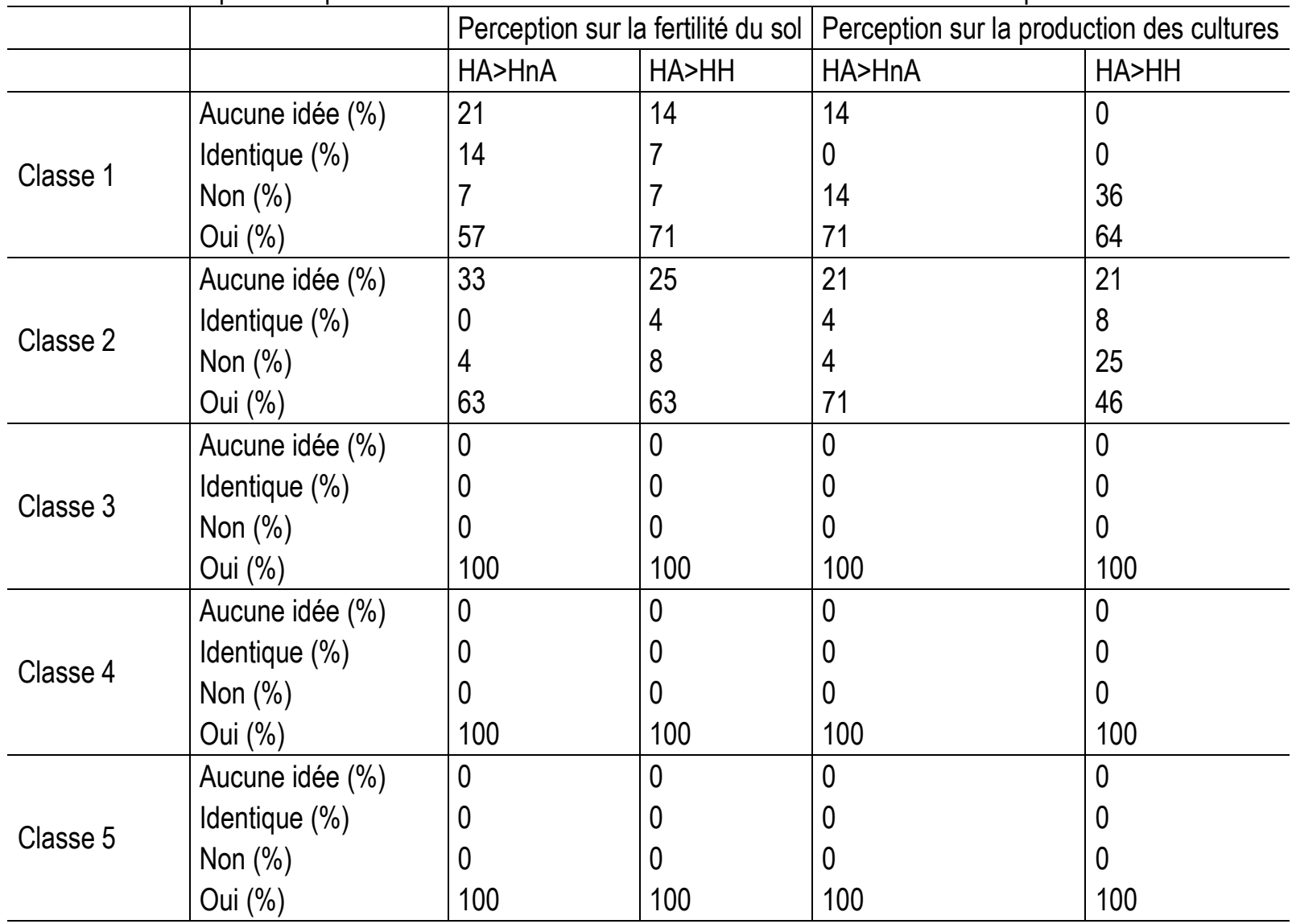

Légende : $H A=$ houppier attaqué, $H n A=$ houppier non attaqué, $H H=$ hors houppier, $>=$ supieur 
Perception des producteurs sur l'utilisation des déjections de chenilles comme fertilisants et sur les modes de collecte et d'application de ces déjections : Les résultats montrent que $71,67,57,67$ et $100 \%$ des producteurs des classes $1,2,3,4$ et 5 respectivement estiment que les déjections de chenilles peuvent être utilisées comme fertilisants du sol (Figure 5). La Figure 6 montre que 43, 33, 14, 33 et $67 \%$ des producteurs des classes $1,2,3,4$ et 5 respectivement, proposent la collecte manuelle des déjections de chenilles. Certains producteurs de la classe $1(7 \%)$ et la classe $2(4 \%)$ proposent de collecter les déjections et les composter. L'option de laisser sur place les déjections de chenilles est proposée par 29, 33 et $33 \%$ des producteurs des classes 3,4 et 5 . Les modes d'application des déjections de chenilles proposés par les producteurs sont l'épandage, l'apport localisé, le mélange avec les engrais chimiques (Figure 7). Le mode d'épandage est proposé par $35,71 \%, 37,5 \%$, $33,33 \%$ et $100 \%$ des producteurs des classes $1,2,4$ et 5 respectivement. Le mode d'apport localisé est proposé par $14,28 \%, 20,83 \%$ et $33,33 \%$ des producteurs des classes 1,2 et 4 respectivement. Seuls les producteurs de la classe 3 proposent de laisser les déjections sur place $(28,57 \%)$ ou de les mélanger avec les engrais chimiques (14,28\%).

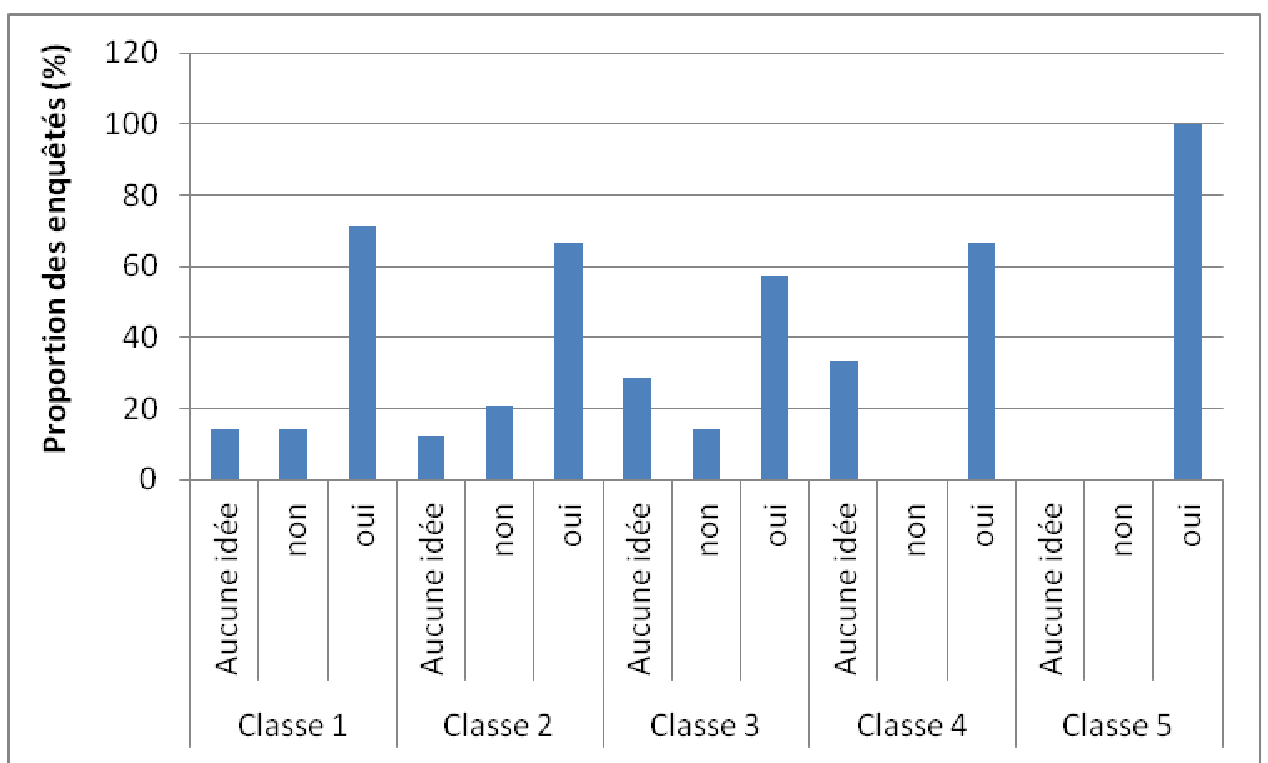

Figure 5 : Utilisation des déjections de chenilles comme fertilisants

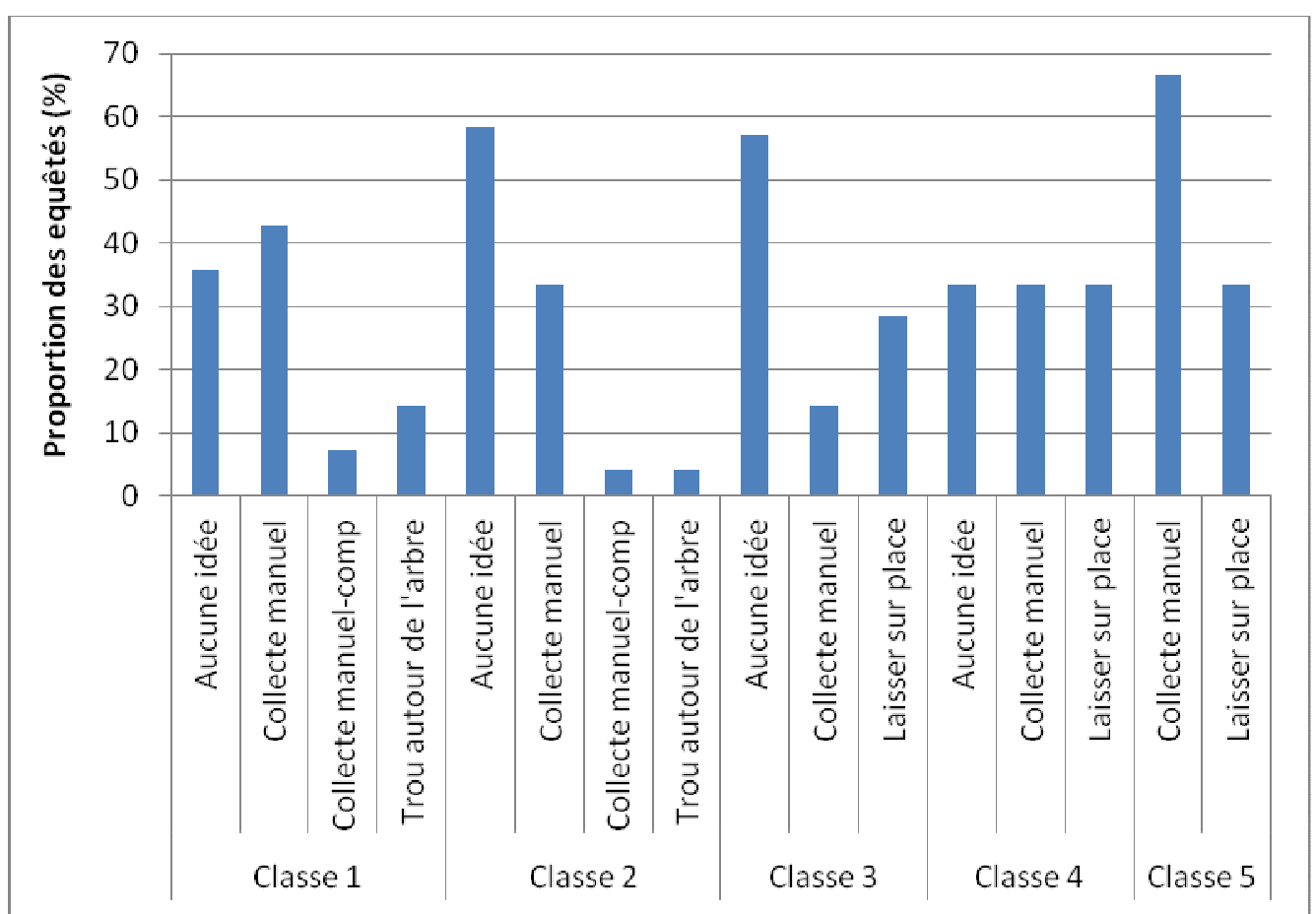

Figure 6 : Modes de collecte des déjections de chenilles proposés par les producteurs 


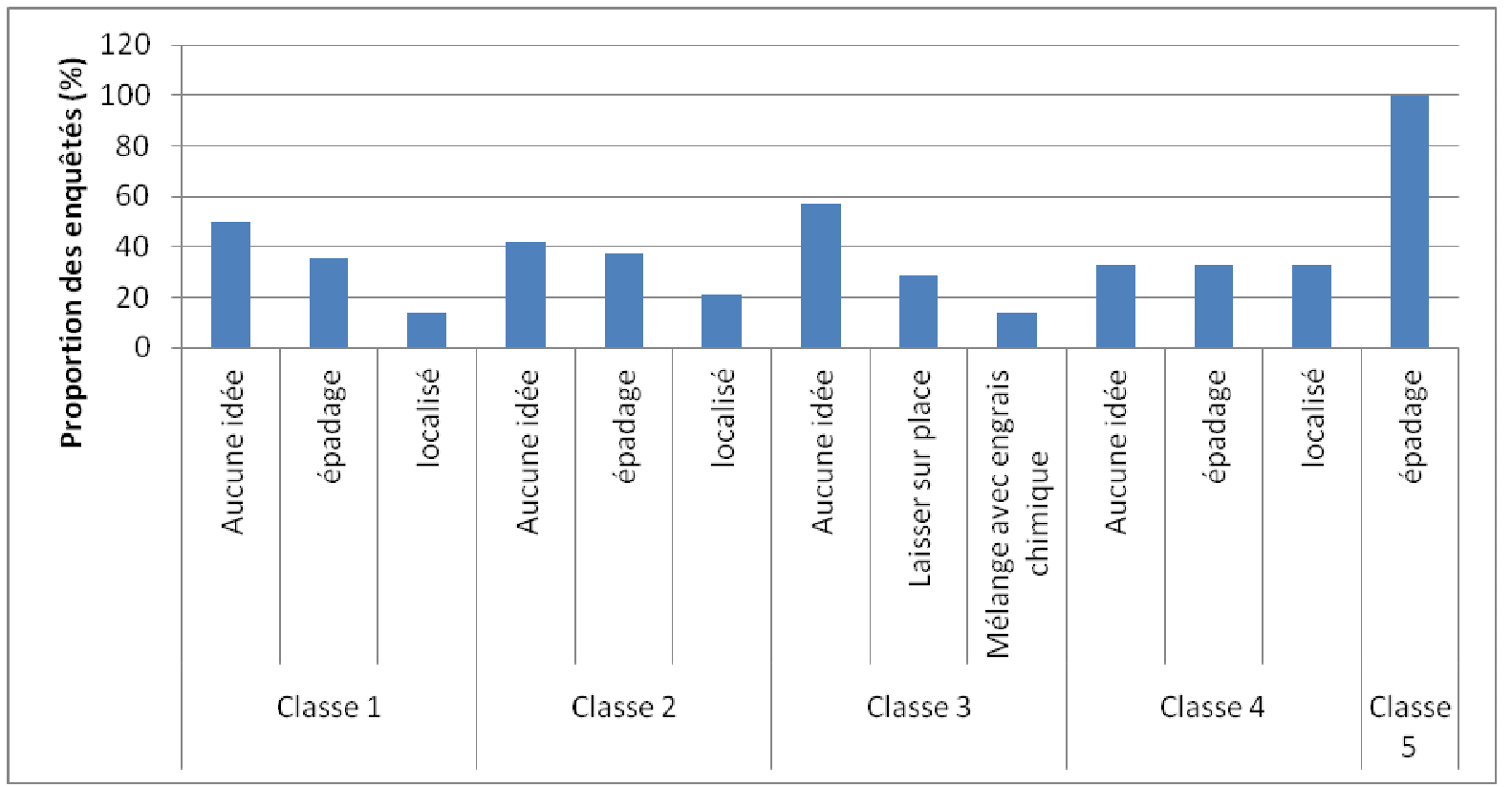

Figure 7 : Modes d'application des déjections de chenille proposés par les producteurs

\section{DISCUSSION}

Les résultats des statistiques descriptives des exploitations indiquent que les producteurs interviewés ont un âge moyen de 42 ans. Ces résultats sur l'âge des producteurs sont similaires à ceux obtenus dans la province du Tuy au Burkina Faso par Blanchard et al. (2014) et au Bénin par Pomalégni et al. (2016). Les données moyennes sur le nombre d'actifs ( 6 actifs) et la surface totale des champs cultivés (12 ha) sont également similaires à celles de Blanchard et al. (2014) qui ont obtenu respectivement 7 actifs et 10 ha par exploitation. Selon Kaboré et al. (2012), les champs dont l'âge de mise en culture est supérieur à 15 ans, sont considérés comme vieux champs. On peut donc qualifier globalement de vieux champs les principaux champs des producteurs enquêtés dontl'âge moyen de mise en culture est de 21 ans. Les résultats sur les classes de producteurs constituées donnent deux classes ( 1 et 5 ) de jeunes producteurs (36 ans), deux classes ( 2 et 4 ) de producteurs d'âge intermédiaire (43 et 42 ans respectivement) et une classe (3) de producteurs âgés ( 59 ans). La classe 3 qui se compose majoritairement de producteurs de la commune de Bama, a la densité de karité la plus élevée. Elle est suivie par la classe 1 qui regroupe également plus de producteurs de Bama. Les autres classes qui regroupent plus de producteurs de la commune de Koumbia, ont de faibles densités de karité. Cela peut s'expliquer par la culture de coton qui est pratiquée dans la commune de Koumbia, ce qui n'est pas le cas dans la commune de Bama. La culture du coton pourrait expliquer également la taille importante des parcelles au niveau de la classe 5 . Les résultats montrent globalement que les chenilles apparaissent chaque année et à la même période selon les producteurs enquêtés. Toutefois, certains producteurs estiment que les chenilles n'apparaissent pas à la même période chaque année. Ils auraient constaté des décalages de temps de deux à trois semaines. Les raisons avancées dans ce cas se sont résumées au début tardif des pluies. La diminution des chenilles au fil des années évoquée par les producteurs serait liée à des facteurs anthropiques, climatiques et édaphiques. Les facteurs anthropiques s'expliquent par une pression de collecte de plus en plus croissante des chenilles au stade larvaire et en début de chrysalisation. Certaines opérations culturales notamment le buttage par le retournement du sol sont responsables de la mort des chrysalides en les exposant au soleil. La coupe des pieds de karité est aussi à l'origine de la baisse des chenilles, parce qu'elle entraine une réduction des peuplements de karité qui constituent l'unique source alimentaire des chenilles selon certains producteurs. L'usage des insecticides lors des traitements de cultures surtout des cotonniers seraient également à l'origine de la mort des chenilles et des chrysalides dans les champs. La plupart de ces observations faites par les producteurs sur les raisons de la baisse des chenilles corroborent les travaux de Son (2007). Selon cet auteur, les opérations culturales telles que le labour, le buttage, I'utilisation de pesticides, sont responsables de la diminution des chenilles. Les facteurs climatiques qui affectent les populations de chenilles selon les producteurs se résument à une irrégularité des pluies. 
Cette irrégularité se ressent à trois niveaux. D'abord à la période d'émergence, une absence de pluies entrainerait un retard dans l'apparition des papillons qui ne peuvent pas émerger du fait de l'état de sécheresse du sol. Ensuite, l'absence ou le manque de pluies lors de la ponte des œufs de papillons inhibe leur éclosion qui nécessite pourtant une certaine humidité. Enfin, après l'éclosion des œufs, l'absence ou le manque prolongé de pluies retarde le développement des larves et les rend vulnérables à la prédation de certaines espèces d'oiseaux. Les inondations des terres occasionnées par de fortes pluies pendant les mois d'août et septembre entraineraient aussi la mort des chrysalides. Les facteurs édaphiques sont liés à la compacité du sol, à certains endroits, qui ne permet pas aux chenilles de s'intégrer dans le sol. Ces observations sur les facteurs édaphiques confirment les travaux de Son (2007) qui précisaient que la faiblesse de la densité de chrysalides dans les zones non cultivées serait liée à la compacité du sol. La perception des producteurs sur la diminution de la population des chenilles est en conformité avec les travaux de Mallet (2013). Cet auteur a montré qu'en zone tempérée, des conditions climatiques notamment le froid et l'humidité influencent négativement sur les populations des coccinelles qui sont pourtant des insectes utiles. Quant à l'impact des chenilles sur la production de karité, la plupart des producteurs ont affirmé que les chenilles ne nuisaient pas à la production de karité. Par contre, d'autres ont déclaré qu'une forte apparition des chenilles sur une campagne de culture engendrait la campagne suivante une baisse de la productivité des pieds de karité. Selon Serpentié (1996), la baisse de la productivité des pieds de karité peut être imputable au parasitisme d'origine animale, ce qui pourrait être le cas des chenilles de karité. Les producteurs ont affirmé majoritairement que les sols sous karité défoliés étaient plus fertiles que les sols sous karité non défoliés et les sols hors houppiers. Les producteurs justifient leur

\section{CONCLUSION}

L'étude que nous avons effectuée visait à analyser la perception des producteurs sur les chenilles de karité et l'effet de leurs déjections sur la fertilité des sols et la production des cultures dans l'Ouest du Burkina Faso. On peut retenir de l'étude que, les producteurs ont identifié les facteurs anthropiques, climatiques et édaphiques comme les facteurs pouvant influencer la dynamique des chenilles suivant les années. II ressort également de cette étude que les déjections contribuent à améliorer la fertilité des sols et à

\section{RÉFÉRENCES BIBLIOGRAPHIQUES}

Bayala J, Mando A, Ouedraogo SJ, Teklehaimanot Z, 2003.Managing Parkia biglobosa and Vitellaria réponse par le fait que les sols sous karité défoliés étaient plus riches en matière organique du fait de l'apport des déjections. Ces sols conserveraient plus d'humidité, ce qui permettrait une décomposition des déjections et les feuilles mortes tombées après la défoliation et donc de disposer d'un stock de matière organique pour l'année suivante. Ces observations des producteurs sur l'amélioration de la fertilité des sols par les déjections viennent confirmer les travaux de Bayala et al. (2003) et Saïdou et al. (2012) selon lesquels les feuilles de karité contribuent à l'amélioration de la fertilité des sols. Les travaux de Coulibaly et al. (2016) montrent également que les déjections de chenilles sont riches en éléments fertilisants avec des teneurs en carbone et en azote de 477,7 et 10,8 g/ $/ \mathrm{kg}$ respectivement. Cette amélioration de la fertilité des sols se traduirait par une amélioration des productions des cultures sous karité défoliés comparés à celles des cultures sous karité foliés et hors houppier. Cette amélioration des productions des cultures sous karité défoliés pourrait s'expliquer également selon les producteurs, par la réduction de l'ombrage due à l'activité des chenilles. Ces observations des producteurs sont en contradiction avec les travaux de Gbémavo et al. (2010) et Seydou et al. (2012) qui ont montré des baisses de production des cultures sous houppiers de karité comparativement aux cultures hors houppiers. La contradiction pourrait se justifier par l'absence de chenilles de karité dans la zone où ces auteurs ci-dessus mentionnés ont effectué leurs travaux. C'est partant du fait que les déjections de chenilles améliorent le niveau de fertilité des sols que la majorité des producteurs enquêtés ont affirmé que les déjections de chenilles pouvaient être utilisées comme fertilisants. Ils ont par conséquent proposé majoritairement la technique de collecte manuelle et l'épandage respectivement comme technique de collecte et mode d'application des déjections.

augmenter la production des cultures selon les paysans. Pour ce faire, les producteurs proposent diverses techniques de collecte (collecte manuelle, creusage de trous de collecte) des déjections et des modes d'apport (épandage, apport localisé). L'utilisation des déjections de chenilles, compte tenu du fait qu'elles sont rejetées à l'échelle des champs, pourrait être envisagée comme mode de gestion de la fertilité des sols en combinaison avec d'autres fertilisants. 
Zone of Burkina Faso, Arid Land Research and Management, 17(3), 283-296.

Blanchard M, Coulibaly K, Bognini S, Dugué P, Vall E, 2014. Diversité de la qualité des fumures organiques produites par les paysans d'Afrique de l'Ouest : quelles conséquences sur les recommandations de fumure ?.Biotechnol. Agron. Soc. Environ. 18(4) : 512-523

Blanchard M, 2005. Relations agriculture élevage en zone cotonnière : territoire de Koumbia et Waly, Burkina Faso. Créteiln Mémoire DESS, Université Paris XII, Val de Marne. $63 p+$ annexes.

Boffa JM, 2000. Les parcs agroforestiers en Afrique Subsaharienne. CIRAFIFAO, $258 \mathrm{p}$.

Coulibaly K, Gomgnimbou APK, Bacye B, Nacro HB, Sedogo MP, 2016. Valorization of shea caterpillar droppings (CirinabutyrospermiVuillet) in the ecological management of soil fertility in Burkina Faso. Int. J. Agri. Agri. R.9(1) : 1-8

Fontès J. et Guinko $S, 1995$. Carte de la végétation et de l'occupation du sol du Burkina Faso. Note explicative. Toulouse : Ministère de la coopération française (France) ; $53 \mathrm{p}$.

Gbémavo DSJC, Glèlè KR, Assogbadjo AE, Katary A, Gnanglè $P, 2010$. Effet de l'ombrage du karité sur le rendement capsulaire du coton dans les agroécosystèmes coton-karité du Nord du Bénin. Tropicultura, 28 (4) 193-199.

Kaboré SA, Bastide B, Traoré S, Boussim JI, 2012. Dynamique du karité Vitellaria paradoxa dans les systèmes agraires du Burkina Faso. Bois et forêt des tropiques, 313 (3), 47-60.

Mallet Z, 2013. Effet des pratiques agricoles sur une communauté d'insectes utiles (les coccinelles), à l'échelle de la parcelle et du paysage. Université de Caen, BasseNormandie, $46 \mathrm{p}$.

Ouédraogo M., 1993. Quelques observations biologiques sur Cirina butyrospermi VUILLET
(Lepidoptera, Attacidea) défoliateur du karité (Butyrospernum paradoxum GAERTN.F) au Burkina Faso. Thèse de Doctorat de 3 ème cycle en Biologie Animale. Université Nationale de Côte d'Ivoire, 123 p.

Pomalégni SCB, Gbemavo DSJC, Kpadé CP, Babatoundé S, Chrysostome CAAM, Koudandé OD, Kenis M,Glèlè KRL, Mensah GA, 2016. Perceptions et facteurs déterminant I'utilisation des asticots dans l'alimentation des poulets locaux (Gallus gallus) au Bénin. J. Appl. Biosci. 98:9330 - 9343

Saidou A, Balogoun I, Kone B, Gnangle CP, Aho N, 2012.Effet d'un système agroforestier à karité (Vittelaria paradoxa c.f. gaertn) sur le sol et le potentiel de production du maïs (Zeamaize) en zone Soudanienne du Bénin. Int. Biol and Chem. Sci., 6(5) : 2066-2082.

Sanon SL, 2005. Valeurs nutritionnelles des « chitoumou ». Communication présentée à la conférence du 11 Août, Bobo-Dioulasso, 5p.

Sermé $B, 2011$. Le circuit de commercialisation et importance économique des chenilles de karité (Cirinabutyrospermii) dans la province du Houet. Mémoire d'ingénieur du développement rural option / Vulgarisation agricole. IDR/UPB, $76 \mathrm{p}$.

Serpentié G, 1996.La production de karité (Butyrospermum paradoxum Gaertn.f. Hepper).Effets de différents modes de gestion des parcs arborés de l'Ouest Burkina Faso. In Amélioration et gestion de la jachère en Afrique de l'Ouest, 73-80.

Son D, 2007. Effets des pesticides sur les insectes non ciblés: cas particulier des chrysalides de Cirina butyrospermii Vuillet dans la zone cotonnière de Pô (Burkina Faso). Mémoire d'Ingénieur du développement rural, option Agronomie. Université Polytechnique de Bobo-Dioulasso, Institut du Développement Rural, Burkina Faso, 68 p. 